\title{
المشكلات اللغوية في تعليم اللغة العربية لدي الطلبة في إندونيسيا
}

\author{
Irsal amin \\ Pendidikan Bahasa Arab IAIN Padangsidimpuan \\ E-mail: aminirsal@gmail.com
}

المادة اللغة العربية لغير العرب مادة صعوبة وغريبة لأن هناك فروق بين اللغة العربية واللغة الأخري إما في

الحرف، والكلمة واللغوية واحوال التعليم. المشكلة الكبيرة التي تواجه الطلبة الإندونسيا في جهة اللغوية لأهما تتعلق عن النفس اللغة ومتعلمها. وأهداف البحث لمعرفة مشكلات تعليمية في ناحية اللغوية التي تواجه الطلبة عند الدرس والتعلم وأحوالها اليومية في تفهم اللغة العربية. هذا البحث هو بحث مكتبي تتعلق عن المشكلات الموجودة في تعليم اللغة العربية لدي الطلبة الإندونسيا. النتائج من الباحث هي مشكلة اللغوية عند التعليم اللغة العربية لغير العرب على خاصة في الأندونيسيا وهي جوانب الاختلاط اللغوي، الجوانب النحوية. المشكلة في جهة اللغة تفريق في بنية الكلمة أن في اللغة الإندونسيا الجملة من Subjek keterangan+objek +prediket ناحية المفردات والكالام وهو كثير في اللغة العربية لاتحاد معادها في الأندونيسيا وما عكسها التي هي شيئ موجودة في العرب ولا في الأندونيسيا. الخلاصة من هذا البحث هو بتجيد المشكلات اللغوية في تعليم اللغة العربية مفاتيح كلمة: مشكلة، لغوية، تعليم
\end{abstract}

\begin{abstract}
Abstrak
Mata pelajaran Bahasa Arab bagi orang Indonesia merupakan mata pelajaran yang sulit dan asing, karena adanya perbedaan antara bahasa Arab dengan bahasa lainnya, baik dari segi huruf, kata, bahasa dan kondisi pembelajaran. Masalah besar yang dihadapi siswa Indonesia dari segi bahasa adalah berkaitan dengan bahasa itu sendiri dan pembelajarnya. Penelitian ini bertujuan untuk mengetahui permasalahan pembelajaran dari aspek kebahasaan yang dihadapi siswa saat belajar dan belajar, dan kondisi keseharian mereka dalam memahami bahasa Arab. Metode yang digunakan dalam penelitian ini adalah metode penelitian kepustkaan. Hasil penelitian ini adalah masalah kebahasaan ketika mengajarkan bahasa Arab kepada orang non-Arab, khususnya di Indonesia, adalah aspek percampuran linguistik, aspek gramatikal. Permasalahan dari segi bahasa adalah perbedaan struktur kata, bahwa dalam bahasa Indonesia kalimatnya berasal dari Subjek + prediket + objek + keterangan, dan dalam bahasa Arab terdiri dari kata kerja + subjek + objek. Perbedaan lainnya adalah dari segi kosa kata dan ucapan, dan dalam bahasa Arab banyak ditemukan kembalinya di Indonesia dan kebalikannya, yaitu sesuatu yang ada di Arab atau di Indonesia.
\end{abstract}

Kata kunci: Masalah, linguistik, pendidikan 


\section{Vol. 9. No. 2 Desember 2021}

اللغة العربية ليست لغة غريبة للاندونيسيين في تعلمها ونطقها، بل أن هذه اللغة من اللغة

الدينية الإسلامية ووجب لتعلمها في تفهيم كتاب اللغة وأحاديث الرسول' . الطلبة في المعاهد

والمدارس يتعلمون اللغة العربية كلغة واجبة لأهما لغة يومية ولغة التدريسي. وكذلك أن المواد

الدراسية كثير باللغة العربية حتي هي لغة مهمة عند الطلبة أن تعلمها وتفهيمها والمادة التعليمية ياللغة العربية؟َ. واللغة العربية هي اللغة واجبة على الطلبة في المعاهد والمدارس لا بتعلها شيى سهول في تعلمها. ووجد فيها مشكلات كثيرة من جهات متنوعة التي تصعب على الطلبة في تدرسيها في نظام الصوت، النحو والصرف والمفردات والأسلوبّ؟. وكذلك جائت في الأفكار الطلبة عند تعليم اللغة العربية يميلو أن هذه اللغة لغة الصعوبة والثقولة حتي تكسلهم في تعلمهاء. تعليم اللغة العربية كلغة أجنبية يختلف عن تعليم اللغة الأم. إن بخاح تعلم اللغة الأم هو بالتأكيد أكثر بجاحًا ويمقق الأهداف، لأن عملية التعلم التي يتم إجراؤها هي عملية تعلم اللغة نفسها، كلغة منطوقة ومكتوبة، بل إها أصبحت لغة التفكير. أصبحت اللغة التي يتم تعلمها عادة بحيث يمكن تطبيقها علميًا على الأشخاص الذين يتعلموها ولا تتطلب سوى التعود 'أحمد سيف الدين, 'تعليم اللغة العربية بالأنشطة اللغوية في مركز نرقية اللغة العربية بمعهد منبع العلوم بتا-

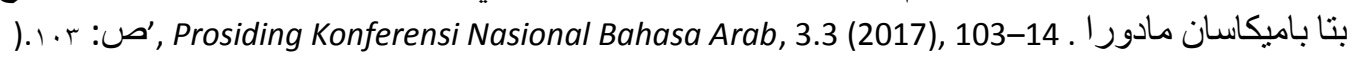
rتامر أمين' and Tamer Amin, اللغويات التربوية ومشكلة اللغة في تعليم العلوم و الرياضيات في العالم

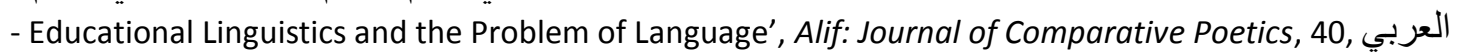
142: ص: 2020, "نور هرينطا, 'مشكلة ترجمة النصوص العربية للطلبة إلمدرسة المتوسطة الاسلامية الحكومية الثانية <http://download.garuda.ristekdikti.go.id/article.php?article=1082661\&val=16375\&title=>.

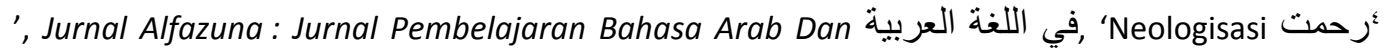
) <https://doi.org/10.15642/alfazuna.v1i1.5>.47 :صKebahasaaraban, 1.1 (2016), 47-78 (p. 


\section{Vol. 9. No. 2 Desember 2021}

وتحسين اللغة لمن يتعلموها. ولنجاح التعليم اللغة العربية فلا بد أن بجعل تعليم هذه اللغة كبرنامج خاصة وهامة من التاج في المدارس الإسلامية وليس من المادة الإضافيةْ.

كانت مشكلة تعلم اللغة العربية كحجر أمام تنفيذ التعلم الفعال حتى الآن لا تزال تبحث دائمًا عن حلولها. أصبحت جوانب مختلفة من مشكلة تعلم اللغة العربية موضوعًا يتم مناقشته دائمًا كوسيلة من وسائل الاجتهاد للخروج من هذه المشكلة. العديد من العروض من مختلف محارسي تدريس اللغة العربية أو الأجنبية لتحسين تعلم اللغة العربية بحيث تتماشى مع لغتها الأجنبي. تتميز مشكلة تعلم اللغة العربية لغير العربية بعدم كفاية أهداف التعلم، حيث لم تكن اللغة العربية قادرة على أن تصبح ضرورة للمجتمع المسلم. تتكون مشاكل تعلم اللغة العربية من مشاكل لغوية تشمل الصوتيات والمفردات والكتابة والصرف والنحو والدلالات.

ومن المعروف لنا أن لغة الإبجيليزية ليس لما المشكلة في تعلمها على الطلبة في أندونيسيا

لأن هذه اللغة مناسبة بلغة الأم يعني لغة الأندونيسيا. اللغة الأندونسيا متاسويا مع اللغة الإبجيليزية إما في قواعدها وصفاتما وكذالك في أساليب تعلم و تعليم. هتان اللغتان كمثل لغة واحدة لا فرق فيها على فرق بعيد التي تصعب في تدرسيها بل اللغة الإنجيليزية لغة سهلة وكثير من اندونسيين يستطعوا أن يتكلمو هذه اللغة ويحبوها حبا شديدا حتى يجعلون هذه اللغة كلغة واجبة وحاجة هامة.

"إسماعيل صو ادي ويككي Kisno Umbar, and Azhar Arsyad, TRADISIONALISME DAN MODERNISME PEMBELAJARAN BAHASA ARAB: PEMBELAJARAN MADRASAH BERBASIS PESANTREN MINORITAS MUSLIM $<$ https://doi.org/10.31227/osf.io/cm257>.10 ص:(INA-Rxiv, 6 November 2017), p. 


\section{Vol. 9. No. 2 Desember 2021}

ولا بد أن تعرف الطلبة في مرحلة والإبتدائية والمتوسطة، والثانوية، والجامعة في مؤسسة

الإسلامية ولو كانوا لا يعرفون شيئا، ولكن اللغة العربية مادة واجبة عليهم فوجب عليهم أن

يتعلمو هذه اللغة. وعلى حسب ذلك، جائت المشكلة للطلبة بمرحلة الجامعة التي لا تتخرجوا من

المدرسة أو المعهد ولا تعرف كثيرا عن اللغة العبية وأحينا منهم لا يعرفوا الحروف الهجائيية ولا

يستطيعوا لكتابتها ولكن يريدوا أن يتكلمو اللغة العربية لأنها مادة واجبة في الجامعة. ل لاهم من

المبتدئين في تعليم اللغة العربية بل أدين منها فمشكلتها في اللغوية تتعلق عن الذات اللغة. الذات اللغة هنا بمعني مشكلة اساسية وموجودة في اللغة ليس في مشكلة التدريس.

ظهرت المشكلة اللغوية في المتعلمين اللغة العربية من جهات متنوعة التي تتصعب في

تعلمها وتطبقها اليومية وتتأثر إلى حماسياةم عند التدريس. المشكلة أصلها من كلمة " " " التي تترجم إلى مشكلة" . أيضا من كلمة "problem" أحوال التي لم تنته وتسبب المسكالت ل . وفي تعريف الأخر هو التناقض بين الرجاء والواقع الذي رجاء لإنتهاء او احتياج أو يعبارة أخري يستطيع أو قليل التناقض ^. المشكلة في فهم اخر هي حالة من عدم الرضا أو التوتر تنشاء عن ادراك وجود عوائق تعترض الوصول إلى الهدف أو توقع

"فوتطر رزقي فطري" ,مشكلات تعلم اللغة العربية وتعليمها لطلاب الفصل السابع في المدرسة المتوسطة الإسلامية

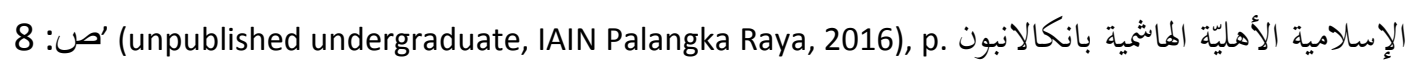
<http://digilib.iain-palangkaraya.ac.id/769/> [accessed 18 May 2021].

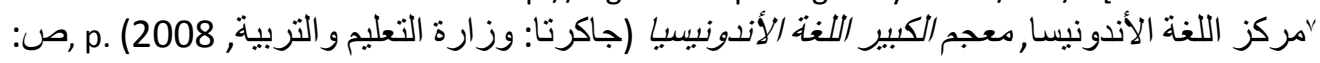

$$
\text { شنوكير, الأساس في إستر/تحية عن الدعوة الإسلامية (سور ابايا: الإخلاص, 1983).p. 65 , }
$$




\section{Vol. 9. No. 2 Desember 2021}

إمكانية الحصول على نتائج أفضل يالإستفادة من العمليات أو الأنشطة المألوفة على وجه حسن أو أكثر كفاية'

وقال الأخر أن المشكلة هي انحراف ما من الحالة الطبيعية إلى مؤثرات سلبية تضر الحالة

النفسية كما تضر البيئة الإجتماعية والإقتصادية والسياسية ويسعي الفرد إلى معرفة أسباب المشكلة لكي يحاول إصلاحها كالمرض ولتسرب والانحراف والجرائم. مثل ذلك: أ) الحالة الضحية للفرد تبقي في حدودها الطبيعية ما لم يؤثر عليها عامل القلق النفس أو عوامل الأمراض الأخري، ب) والحالة الإجتماعية للمجتمع والفرد تبقي في حدودها الطبيعية'.' قال محمد عينين ووركون مالباري أن المشكلة في تعليم اللغة العربية تنقسم إلى قسمين وهما مشكلة في العوامل اللغوية والعوامل غير اللغوية ويقع أيضا في المهارة الأربعة. العوامل اللغوية وهي العوامل التي تعود إلى اللغة نفسها أي العلوم الموجودة فيها. وهذا تظهر إلى الأصوات والمفردات والقواعد النحوية والصرفية. والعوامل غير اللغوية هي يندرج تحت هذه العوامل التي ليست لها علاقة بطيبعة اللغة إلا أها تؤثر في تعليم اللغة بشكل مباشرة وفعال (صالح). وهذه "جممد إيصام الددين' ,مشكلات تعليم اللغة العربية في مدرسة مغتاح العلوم الثانوية بكالسات جمبر وحلها '

ص: unpublished undergraduate, Universitas Islam Negeri Maulana Malik Ibrahim, 2015), p. <http://etheses.uin-malang.ac.id/8937/> [accessed 18 May 2021].

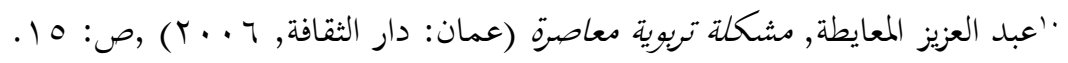




\section{Vol. 9. No. 2 Desember 2021}

العوامل تتكون من عدة المشكلة، إما من ناحية الطلبة والمدرس وإما من ناحية الطريقة أو البيئة 'ا

وأما المشكلة التعليمية للطلبة الأندونيسيا في كل المرحلة أن هناك مشكلتين رئيسيتين تتعلقان بتعليم اللغة العربية، وهما تتمثل في المشكلة اللغوية وغير اللغوية. واما المشكلة اللغوية فتحتوي الصوتي، والمستوي النحوية والكتابة في الإملاء بسبب صعوبة في فهم القواعد'. المشكلة الصوتية: بند أن تعليم العربية بأندونيسيا لم يعتن بهذا المحال. وقد جائت عناية تعليم العربية منذ زمن بعيد مازال ولم يزل قائمة على جانب القراءة. فالقراءة كما هو معروف لم تعتن بالناحية الصوتية بكثرة. والمشكلة الإخري من الجهة الصوتية تواجه الطلبة بالأندونيسيا أن فيها أصوات لم يكن هناك مقابل في الأصوات الأندونيسيا حتي تكون الصعبة في نطق عند الكلام لأن تختلف عن اللغة الأمب" . الحروف التي لم بحد في اللغة العربة للإندونسيا، مثل :

\begin{tabular}{|c|c|c|c|c|c|c|c|c|c|c|}
\hline$\dot{\varepsilon}$ & ف & $\varepsilon$ & ظ & b & ص & ض & ذ & $\dot{\tau}$ & $\tau$ & ث \\
\hline ghain & pha & 'ain & Zhã & Tha' & shãd & Dhãd & dzã & khâ & hâ & Tsâ \\
\hline
\end{tabular}

" حوميدى، عدد المشكلات وظهورها وحلولها في تعليم اللغة العربية:دراسة ميدانية بجامعة الإسلامية شيخنا محمد

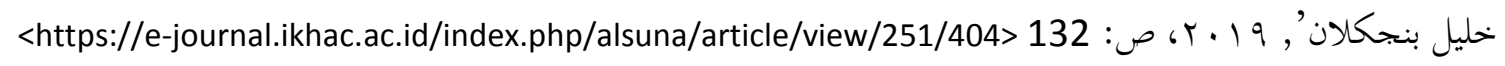
[accessed 18 May 2021].

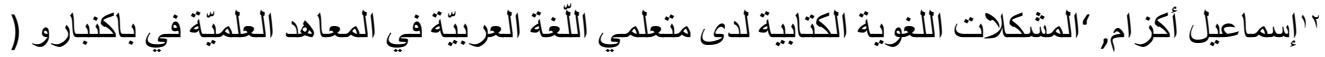

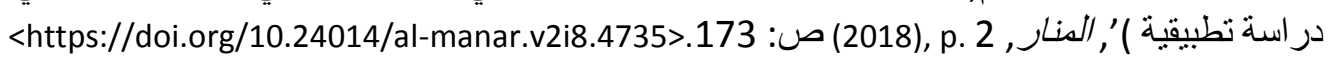

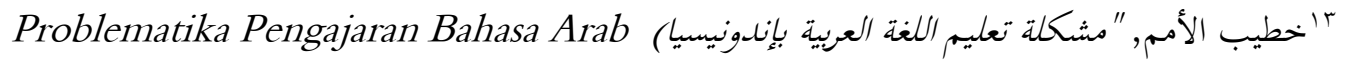
" Di Indonesia العدد 1 ,ص: -0-11. 


\section{Vol. 9. No. 2 Desember 2021}

والحروف العربي التي لمج تجد في اللغة الأندونيسيا تسبب الصعبة عليهم في النطق أحروفها، والأحروف السابقة تتغير عند الكلام لغير العرب. والتغير في ذلك النطق للكلمات التي أصلها من اللغة العربية كمثل كلمة الظهر تتغير إلى لوحور (luhur atau lohor،zuhur) . التغيير في هذه الكلمة تغير من الحرف "ج" إلى الحرف "ذ" وعند النطق لاتناسب على معنى الأصل.

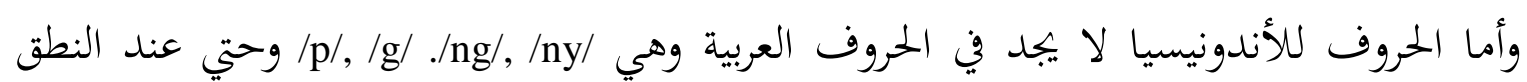
تتغير إلى الحرف الأخر كمثل في النطق /pepang تكون /b/p/ مثلهون اليبان وحرف /g/ تكون "غ" او "ج" كمثل Spanyol أن تكون اسبانياء .

المشكلة اللغوية من الناحية المفردات تتكون من عدة العوامل: أ) إقبال كبير من المفردات اللغة الأندونيسيا. فهناك مفردات إندونسيا ضخمة أصلها مفردات عربية حتي يقول أحد الباحثين الهولنديين أن نسبة المفردات الإندونيسيا مأخوذة من العربية لا تقل على سبعين بالمائة. ويالرغم من ذلك مشكلات في تغيير معاني هذه المفردات. فكلمة "مشاركة" في العربية تعني /المساهمة/ أما في الأندونيسيا فمعناها /البمتمع، كلمة "دوان" في العربية معناها إدارة وفي الأندونيسيا معناها Dewan. ب) اللفظ تتغير من أصله، كلمة "البراكة" تكون berkat، "الخبر" تكون kabar، "يمكن" تكون mungkin، "موافقة" تكون mufakat، ج) اللفظ الثابت ومعناه تتغير ومثل الكلمة التي تدل إليها هو "كلمة" في العربية هي المفردات ولكن في الأندونيسيا تتكون من عدة المفردات التي تسرح وتعطي التعريفات. ؛ أجيف حرماو ان, طريقة التعليم اللغة العربي (بندونغ: رشد كريا, 2018) p. ,ص: 112. 


\section{Vol. 9. No. 2 Desember 2021}

المشكلة في جهة الصرفية هي تغيير الكلمة مناسبة على أسلوب الصرف من كلمة إلى

كلمة في الفعل والأسم. الفعل يتغير إلى الاسم والإسم يتغير إلى الفعل وتغير معان الكلمة. وهذا التغيير سمي في اللغة العربية "تصريفية" (konjungsi). التغيير في الفعل يشتمل إلى المذكر والمؤنث وتغيير اخر تتعلق إلى فاعله ومواصه في الجملة حتي تكون فيها المعان الكثيرة. وعلى سبيل المثال

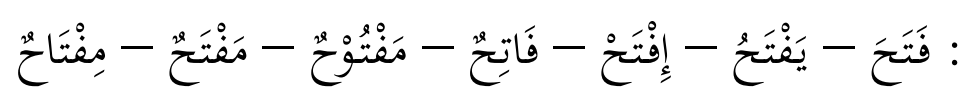

\begin{tabular}{|c|c|c|}
\hline | فعل ماض & Telah membuka & فنتَّحَ \\
\hline فعل مضارع & Sedang membuka & بَفْتَحُ \\
\hline فعل الأمر & bukalah & فِقتَحْ \\
\hline فاعل & Orang yang membuka & فَاتِعَّ \\
\hline | مفعول به & Yang di buka & تَفْتُوُْحُ \\
\hline | إسم المكان & Tempat membuka & فَفْتَحُ \\
\hline إسم الألة & Alat pembuka & فِفْتَاحُ \\
\hline
\end{tabular}

الكلمات السابقة هي تغير الكلمة في اللغة العربية التي تقابل الطلبة للإندونيسيا بلغتهم حتي تجدوا كثرن من الصعبة في التعليم والتدريب للكلام والكتابة. المدرس اللغة العربية عند التعليم تجد الصعبة لتفهم الطلبة على تغيير الكلمات ويتأثر في الكتابة وفي معاها. الطلبة في تفهيم المواد الدراسية تتعلق عن الصرف أحينا يأحذ من اللغة الأندونيسيا ثم يبحثوا موازينها في العربية وتصعبهم لأن اللغة العربية والأندونيسيا لا تساوي مع العربية في اللغة وفي تغيير الكلمة. وعلى نظر الفعل في العربية هناك فعل الماض والمضارع والأمر وتصريفه لكل الفعل لديه تغيير الخاصة لكل الأفعال الموجودة، وفي الأندونيسيا تتفرق عنها حتي لاتؤدي معانيها وتصعب في 


\section{Vol. 9. No. 2 Desember 2021}

فهمها لغير العرب. وكذلك في تعديد الكلمة الأندونيسيا بالمفرد والجمع ولكن في العربية الكلمة تتكون من المفرد والمثني والجمع، وفي الجمع هناك المذكر السالم والمؤنث السالم وجمع التكثير. ومن المشكلة النحوية بند مشكلة الإعراب والبناء تحول دون الطلبة، وهذا الأن يستخدم في المعاهد والمدارس وحتي في الجحامعات على النحو التقليدي بما فيه العوامل والأقيسة والأراء المنطقية وبجانب ذالط هناك نظام المطابقة التي لم يجدها في لغتهم الأم. وعلى حسب هذه المشكلة تظهر مشكلة الكتابة لأن عند الكتابة تحتاج معرفات كثيرة عن النظام النحوي لكتابة الجملة في مكاها المناسبة حتي تكون الجملة كجملة مفيدة. من غير مشكلة في اللغوية هناك مشكلة أخري تتعلق بالمهارات اللغوية تعد أصعب لدي الطلبة في تعليم اللغة العربية إما في مهارة الإستماع والكلام والقراءة والكتابة. وللمهارة اللغوية تحتاج إلى تعويد وتدريب الدارسين على تطبيق اللغة العربية في بيئتها الأصلية وربما في نشأة اللغة ومراعتها ومرافقها في استعانة المدرس في تكيف حالتهم اليومية.

وأما المشكلة غير اللغوية على الطلبة في ناحية ثاقفية واجتماعية، الثقافة العربية تختلف عن الثقافية الإندونيسيا وبلد أخر من غير العرب وكذالك في مشكلة الطريقة التعليمية لم تكن شاملة ولاتزال من ناحية التقليديْ1. وهذا الجانب يمكن إداركه حينما يتعلم الطلبة العبارات العربية فقد كانت وراء هذه العبارات عادة وتقاليد وظروفهم الخاصة. فهناك عبارة "قبل الرماء 


\section{Vol. 9. No. 2 Desember 2021}

تملاء الكنائن" فهذه العبارة قائمة على بيئة عربية كثرت فيها الغزوات والحروب وفي الأندونيسيا فلم تعتد الحروب وتكثر فيها الأمطار والعبارة الاندونيسيا فيها العبارة الخاصة أيضا لا تحد في دول العربية. ومن هذه الحالة لابد للمدرسين اللغة العربية في الأندونيسيا ان تعلموا الثقافة العربية الإسلامية التي لها أثار وأعمال متوافرة من الكتب والمؤلفات المتنوعة. وينبغي ان لاتنسى على المشكلات والعوائق التي تصدر من اغراض المتعلمين وبتطور العلوم والتكنولوجيا أصبح تعلم اللغة العربية السهولة عند عصرنا العولمة.

\section{طريقة البحث}

هذا البحث هو بحث مكتبية تتعلق عن المشكلات الموجودة في تعليم اللغة العربية لدي الطلبة لغبر الناطقين بها وأهدافها لمعرفة مشكلات تعليمية في ناحية اللغوية التي تواجه الطلبة عند الدرس والتعلم وأحواها اليومية في تفهم اللغة العربية. واما الطريقة في جمع البيانات باستخدام طريقة الدراسة التوثيقية على الكتب والمحلات العلمية تتعلق عن هذه المشكلة. بعد وجد البيانات فحلل البيانات بطريقة تحليل الضمن على البمالات المتعلقة بمشكلة تعليمية التي واجهة الطلبة لغير الناطقين بها. نتائج البحث الاختلاف في الكلمات بين اللغة الاندونيسية واللغات الملايو الأخرى في المفردات، حيث تتكون المفردات العربية في بعض العبارة كلمتين ومعناها واحد. بينما في اللغة الإندونيسية كلمة واحدة لها معنى واحد. بالإضافة إلى ذلك، يعتبر نقل اللغة العربية إلى الإندونيسية مشكلة 


\section{Vol. 9. No. 2 Desember 2021}

بحد ذاتا للطلاب الإندونيسيين الذين يدرسون اللغة العربية بحيث تصبح عقبة وهناك افتراض بأن اللغة العربية لغة صعبة. نشأ هذا الافتراض في دوائر مختلفة، من الطلاب والمعلمين، ولم يتم حله لأغم لم يفهموا الفرق بين اللغة التي يتم تدريسها واللغة المستهدفة. من بين المشاكل اللغوية بين العربية والإندونيسية أو غيرها من لغات الملايو من عدة جوانب: أ) جوانب الاختلاط اللغوي، الأول: العديد من الكلمات في اللغة الإندونيسية مأخوذة من اللغة العربية أو أن الكلمات الأساسية مأخوذة من اللغة العربية بحيث يكون هناك تحول في المعنى في الإندونيسيين العاديين. في اللغة الإندونيسية، كلمة بجتمع تشبه كلمة مشاركة، بينما إذا قمنا بترجمتها إلى العربية، فإن كلمة بجتمع هي وحدة. وتعني كلمة "البحلس" المجلس هو المكتب الذي يعني اللغة العربية. أفضل ما في الأمر هو أن هذه المقالة نُشرت في الأصل باللغة الإندونيسية. الثاني: تغيّر اللفظ عن الصوت الأصلي، مثل كلمة بركة من كلمة "بركة"، والكلمة من كلمة "خبر"، وربما من كلمة "ربما" من كلمة "موفقة". أفضل ما في الأمر هو أن هذه المقالة نُشرت في الأصل باللغة الإندونيسية. الثالث: النطق ثابت لكن المعنى يتغير، مثل الجملة اللفظية التي تعني تركيبة الكلمات التي يمكن أن تعطي معنى، تأتي من اللغة العربية والتي تعني الكلمات، ب) الجوانب النحوية، هناك فرق كبير بين العربية والإندونيسية والإنحليزية. توجد أوجهه تشابه بين اللغتين الإندونيسية والإنحليزية بحيث يجد الإندونيسيون تعلم اللغة الإنحليزية أسهل من تعلم اللغة العربية لأن قواعد اللغة الإندونيسية والإنحليزية هي نفسها، أي اتباع نمط الموضوعات والتنبؤات والأشياء والكلمات. لفهم اللغة الإنحليزية، بالطبع، سيكون من الأسهل إذا كانت الأنماط والأشكال 


\section{Vol. 9. No. 2 Desember 2021}

متماثلة، ما عليك سوى البحث عن المعنى. وفي الوقت نفسه، لفهم اللغة العربية، بالإضافة إلى الاضطرار إلى معرفة معنى كل جملة، يجب على الطلاب أيضًا فهم قواعد اللغة العربية، بحيث يكون الجهد الذي يتعين عليهم القيام به أكثر من تعلم اللغة الإنحليزية.

تعرف قواعد اللغة العربية بالقواعد النحوية التي تدرس قواعد اللغة العربية كدليل في قراءة الكتب العربية وفهم الجمل العربية. ستوجه القواعد النحوية الأشخاص في قراءة وفهم جملة باللغة العربية لتجنب الفهم المختلف وحتى الخاطئ للجملة أو النص في اللغة العربية. عادة، الكتاب اللغة العربية تكتب بدون الحركات لأهما تعتبر قادرة على قراءمّا بناء على القواعد النحوية في

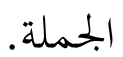

هناك اختلاف كبير جدًا بين اللغة العربية واللغات الأجنبية الأخرى مثل الإندونيسية والإنحليزية هو بنية الجملة. في اللغة الإندونيسية أو الإنحليزية، تتكون بنية الكلمة من keterangan+objek +prediket +Subjek بينما في اللغة العربية تتكون بنية الكلمة من fl'il + fail +objek+keteranga+ من المؤكد أن الاختلاف في بنية الكلمات بين العربية والإنحليزية أو الإندونيسية هو اختلاف يجعل الطلاب الذين يتعلموها يشعرون بصعوبة لأن عليهم تذكر هذه الاختلافات بالترتيب قبل نطقها أو كتابتها. في هذا الترتيب المختلف، بالطبع، لا يزال في دراسة بسيطة، ناهيك عن فحص الكلمات التي لها الحق في أن تصبح موضوع الكلمة وخبرها وموضوعها. في اللغة العربية، بالطبع، عليك أن تناقش فيل، ففيل ومافولومبل أولاً قبل تكوين بنية جمل مثالية. المناقشة بالطبع يجب أن تدرس 


\section{Vol. 9. No. 2 Desember 2021}

الكلمات مقدمًا والخصائص التي تتوافق مع الكلمات المقصودة حتى لا تكون هناك أخطاء في تحضيرها. أفضل ما في الأمر هو أن هذه المقالة نُشرت في الأصل باللغة الإندونيسية. بشكل عام، تنقسم مشكلة تعلم اللغة العربية من الناحية النحوية إلى نوعين من المشاكل، وهما: أ) المشاكل المورفولوجية، وهي مشكلة أشكال الكلمات (Sighot) التي تتبع نمط تغيرات الكلمات في النظام الصرفي / الكلمات الهجينة الموجودة في اللغة العربية والتي تختلف بالتأكيد عن اللغات الأجنبية الأخرى. التغييرات في شكل الكلمات في لغة اللغة تتبع التغييرات الواردة في الفصل وموضوع التغيير (شرف)، والذي يحتوي كل فصل على نمط تغيير وقواعد معينة. في دراسة شرف باللغة العربية هناك تغييرات خاصة لمعرفة احتياجات الكلمة وتغييراتما. أفضل ما في الأمر هو أن هذه المقالة نُشرت في الأصل باللغة الإندونيسية، ب) مشاكل نحوية/نوية، هذه المشكلة هي مشكلة ترتيب الكلمات التي تأخذ في الاعتبار العلاقة بتقنية ترتيب الكلمات العربية بعد فهم نظام الصوت. هذه المشكلة ستجعل الأمر صعبًا على الطلاب بسبب الاختلافات بين تكوين اللغة الأم واللغة العربية. توضح العديد من الدراسات أن الطلاب يجدون صعوبة في تعلم قواعد اللغة العربية. من بين المشاكل الموجودة في تعلم اللغة العربية لغير العرب، تعد مشكلة ترتيب الكلمات هذه من أكبر المشاكل التي تربك متعلمي اللغة العربية أنفسهم. بالإضافة إلى مشكلة المتعلمين، فهي أيضًا مشكلة بالنسبة لمعلم اللغة العببة، فمعلم اللغة العربية نفسه يشعر أحيانًا بمدى صعوبة تعليم فهم قواعد اللغة لطلابه 
بناءً على التحليل في هذه الدراسة، يمكن الاستنتاج أن مشاكل تعلم اللغة العربية للإندونيسيين وغيرهم من مستخدمي اللغة هي الاختلافات في هوية اللغة. الفرق بين الاثنين هو من حيث المفردات مع الكلام الذي ليس له معادل، كثير في اللغة العربية لاتحد معادها في الأندونيسيا وما عكسها التي هي شيئ موجودة في العرب ولا في الأندونيسيا، وأما في القواعد بسبب الاختلافات في بنية الجملة، من حيث لم تتغير الكلمات في اللغة الإندونيسية بينما تغيرت اللغة العربية، على سبيل المثال أن في الأندونيسيا في كتابة menulis لفعل المضارع لابد بكتابة sedang وأما في العربية بفعل المضارع أن القارئين الفاهمين، و من حيث التدريس كثير من مدرسي اللغة العربية. لهذا الاختلاف تصبح مشكلة في تعلم اللغة العربية. 
Fitri, Putri Rizki, 'مشكلات تعلم اللغة العربية وتعليمها لطلاب الفصل السابع في المدرسة 'المتوسطة الإسلامية الأهليّة الهاشمية بانكالانبون (unpublished undergraduate, IAIN Palangka Raya, 2016) <http://digilib.iain-palangkaraya.ac.id/769/> [accessed 18 May 2021]

Humaidi, 'View of: عدد المشكلات وظهورها وحلوها في تعليم اللغة العربية:دراسة ميدانية r بhttps://ejournal.ikhac.ac.id/index.php/alsuna/article/view/251/404> [accessed 18 May 2021]

Isomuddin, Moh, ، مشكلات تعليم اللغة العربية في مدرسة مفتاح العلوم الثانوية بكالسات جمبر 'وحلها (unpublished undergraduate, Universitas Islam Negeri Maulana Malik Ibrahim, 2015) <http://etheses.uin-malang.ac.id/8937/> [accessed 18 May 2021]

أجيف حرماوان, طريقة التعليم اللغة العربي (بندونغ: رشد كريا, 1 | • ب)

أكزام, إسماعيل, "المشكلات اللغوية الكتابية لدى متعلمي اللّغة العربيّة في المعاهد العلميّة في

rhttps://doi.org/10.24014/almanar.v2i8.4735>

اللغويات التربوية ومشكلة اللغة في تعليم العلوم والرياضيات في ' and Tamer Amin, أمين, تامر - Educational Linguistics and the Problem of Language', Alif: Journal of Comparative Poetics, 40, 2020, I $\leqslant r_{-}$I $7 \mathrm{~V}$

الدين, أحمد سيف, "تعليم اللغة العربية بالأنشطة اللغوية في مركز ترقية اللغة العربية بمعهد منبع 'العلوم بتا-بتا باميكاسان مادورا ,', Prosiding Konferensi Nasional Bahasa Arab, 3.3 (2017), 103-14 
Thariqaah Ilmiyah; Jurnal Ilmu-IImu Kependidikan dan Bahasa Arab

\section{Vol. 9. No. 2 Desember 2021}

(Problematika Pengajaran Bahasa Arab Di Indonesia)" جاكرتا: كلية الآداب - جامعة شريف هداية الله الحكومية) الإسالامية, 9999 ( ), دورية : التراث، العدد م

في اللغة العربية Nurnal Alfazuna : Jurnal Pembelajaran Bahasa Arab Dan Kebahasaaraban, 1.1 (2016), 47-78 <https://doi.org/10.15642/alfazuna.v1i1.5>

شوكير, الأساس في إستراتجية عن الدعوة الإملامية (سورابايا: الإخلاص, بم 1 )

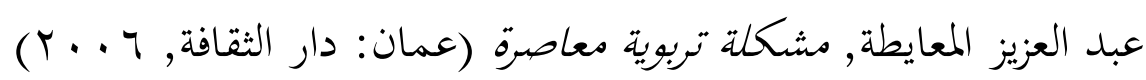

,' TARBAWY : Indonesian Journal of Islamic Education, 7.2 (2020), 219-28 <https://doi.org/10.17509/t.v7i2.25593>

مركز اللغة الأندونيسا, معجم الكبير اللغة الأنلدونيسيا (جاكرتا: وزارة التعليم والتربية, م · . ب) نور هرينطا, "مشكلة ترجمة النصوص العربية للطلبة الملدرسة المتوسطة الاسلامية الحكومية الثانية 'بنجكولو <http://download.garuda.ristekdikti.go.id/article.php?article=1082661\&val=16375 \&title=>

, ويكككي, Kisno Umbar, and Azhar Arsyad, TRADISIONALISME DAN Modernisme Pembelajaran Bahasa Arab: Pembelajaran Madrasah Berbasis Pesantren Minoritas Muslim (Ina-Rxiv, 6 November 2017) 